\title{
FORC Study of Magnetization Reversal and Interlayer Interactions in Rapidly Quenched Fe/Co-Based Bilayer Ribbons
}

\begin{abstract}
B. KUnCA ${ }^{a *}$, I. MAŤKO ${ }^{b}$, P. ŠveC ${ }^{b}$ AND I. ŠKORVÁNEK ${ }^{a}$
${ }^{a}$ Institute of Experimental Physics, Slovak Academy of Sciences, Watsonova 47, 04001 Košice, Slovakia

${ }^{b}$ Institute of Physics, Slovak Academy of Sciences, Dúbravska Cesta 9, 84511 Bratislava 45, Slovakia

Study of the interlayer interactions and hysteresis processes was performed for the nano/microcrystalline bilayer ribbon composed of soft magnetic $\mathrm{Fe}_{73.5} \mathrm{Cu}_{1} \mathrm{Nb}_{3} \mathrm{Si}_{13.5} \mathrm{~B}_{9}$ layer and semihard magnetic $\mathrm{Co}_{72.5} \mathrm{Si}_{12.5} \mathrm{~B}_{15}$ layer. $_{2}$. Precursor amorphous ribbons were prepared by modified double-nozzle planar flow casting method. Measurement of the First Order Reversal Curves (FORCs) was utilized for detailed characterization of its magnetic hysteresis behaviour. Calculated switching field distribution (SFD) consists of two distinct peaks, each representing specific magnetic phase. With decreasing reversal field value, SFD peak of the microcrystalline, semihard magnetic Co-based layer is shifted to the higher field values, indicating presence of strong positive exchange interaction. On the other hand, SFD peak of the soft magnetic nanocrystalline Fe-based layer is shifted to lower and even negative field values. Such behaviour was associated with the presence of magnetostatic bias field originating in the semihard magnetic layer.
\end{abstract}

DOI: 10.12693/APhysPolA.137.815

PACS/topics: nanocrystalline alloys, bilayer ribbons, magnetic hysteresis, magnetostatic interaction

\section{Introduction}

Rapidly solidified amorphous or nanocrystalline bilayer ribbons prepared by rapid quenching using the modified double-nozzle technique [1] are subject of ongoing interest to both physicists and technologists due to the possibility to combine different magnetic and/or magnetoelastic properties of constituting ferromagnetic phases in one system [2-4]. Better understanding of its hysteresis response to the external magnetic field and unveiling of the internal magnetic interactions may be achieved by First Order Reversal Curve (FORC) method. A great deal of attention has been dedicated to FORC analysis of permanent magnets $[5,6]$, or thin film bilayer systems $[7,8]$. However, considerably less attention has been given to bilayer systems in ribbon form. In this work, FORC analysis of the magnetization reversal processes and interlayer interactions was conducted for ferromagnetic $\mathrm{Fe}_{73.5} \mathrm{Cu}_{1} \mathrm{Nb}_{3} \mathrm{Si}_{13.5} \mathrm{~B}_{9} / \mathrm{Co}_{72.5} \mathrm{Si}_{12.5} \mathrm{~B}_{15}$ bilayer ribbon. Mutual influence of soft and semihard magnetic layers on magnetization reversal in this bilayer system was studied by means of switching field distribution (SFD), as well as FORC distribution.

\section{Samples and methods}

Rapidly quenched bilayer ribbon with average thickness of $38 \mu \mathrm{m}$ consisted of $\mathrm{Fe}_{73.5} \mathrm{Cu}_{1} \mathrm{Nb}_{3} \mathrm{Si}_{13.5} \mathrm{~B}_{9}$ layer (air side of the ribbon) and $\mathrm{Co}_{72.5} \mathrm{Si}_{12.5} \mathrm{~B}_{15}$ layer (wheel

*corresponding author; e-mail: kunca@saske.sk side of the ribbon) and has been prepared by the planar flow casting from a single crucible using the modified double-nozzle technique [1]. Thermal analysis of prepared samples was conducted by differential scanning calorimetry (DSC) at the heating rate $10 \mathrm{~K} / \mathrm{min}$. According to these data, the as-quenched ribbons were isothermally annealed under high vacuum for $180 \mathrm{~s}$ at $843 \mathrm{~K}$. Changes of microstructure upon annealing were investigated by cross-sectional transmission electron microscopy (CS-TEM) and selected area electron diffraction (SAED) using JEOL 2000FX at $200 \mathrm{kV}$. First Order Reversal Curves (FORCs) of $2 \mathrm{~mm}$ wide and $6 \mathrm{~mm}$ long annealed bilayer sample were obtained using the MicroSense EV9 vibrating sample magnetometer (VSM) between saturating magnetic field of 1000 Oe and reversal fields ranging from 100 Oe to negative value of saturation field. Switching field distribution (SFD) was calculated for each FORC as a derivative of measured magnetization with respect to the applied field

$$
\operatorname{SFD}\left(H, H_{r}\right)=\frac{\partial M\left(H, H_{r}\right)}{\partial H} .
$$

FORC distribution has been acquired as a mixed secondorder derivative of magnetization with respect to applied field $\mathrm{H}$ and reversal field $H_{r}[9]$ :

$$
\rho_{\mathrm{FORC}}=-\frac{\partial^{2} M\left(H, H_{r}\right)}{\partial H \partial H_{r}} .
$$

Grid of $M\left(H, H_{r}\right)$ points was specified using a smoothing factor $S F=3$. Value has been chosen on the basis of estimation of standard deviation proposed in [10]. Results are plotted as a $2 \mathrm{D}$ contour plot using coordinates $\left(h_{c}, h_{u}\right)$, which refer to critical fields $h_{c}=\frac{1}{2}\left(H-H_{r}\right)$ and interaction fields $h_{u}=\frac{1}{2}\left(H+H_{r}\right)$. 


\section{Results and discussion}

Figure 1 shows thermal analysis and microstructure development in the studied bilayer ribbon. DSC thermograph of the studied bilayer sample (Fig. 1a) shows that crystallization temperatures of the bilayer ribbon well correspond to the ones of the reference singlelayer ribbons, prepared by conventional planar flow casting method. The first crystallization event observed for the $\mathrm{Fe}_{73.5} \mathrm{Cu}_{1} \mathrm{Nb}_{3} \mathrm{Si}_{13.5} \mathrm{~B}_{9}$ layer with onset at at $T_{x 1}=783 \mathrm{~K}$. and peak position at $T_{p 1}=809 \mathrm{~K}$ corresponds to the formation of the nanocrystalline bcc-Fe(Si) grains typical for FINEMET alloys [11]. These events could be recognized in the upper part of cross-sectional CS-TEM image in Fig. 1b. Grain sizes of $12-15 \mathrm{~nm}$ were determined from XRD measurements using the Bruker D8 Advance $\left(\mathrm{CuK}_{\alpha}\right.$ radiation) with Goebel mirror in the incident beam and LiF monochromator in the diffracted path, and detailed higher magnification TEM images (not included). Crystallinity content was estimated to $\approx 50-60$ vol. $\%$ of this layer. Primary crystallization in the $\mathrm{Co}_{72.5} \mathrm{Si}_{12.5} \mathrm{~B}_{15}$ layer begins above $\sim 825 \mathrm{~K}$ and is associated with formation of the microcrystalline grains of Co-rich phase, observable in the lower part of the same CS-TEM image. Further crystallization of its amorphous matrix occurs above $856 \mathrm{~K}$. Relatively low degree of intermixing between the individual layers can be observed in the close vicinity of their common interface, which is of planar character and its thickness lies below $500 \mathrm{~nm}$. The CS-TEM image of the interlayer region is enclosed by selected area electron diffraction (SAED) patterns of its regions.

Switching field distribution (SFD) curves shown in Fig. 2, were calculated from measured set of First Ordered Reversal Curves. They show a two-step magnetization process that is characteristic for bi-phase magnetic systems. Sharp peak in the low magnetic field region corresponds to the nanocrystalline soft magnetic $\mathrm{Fe}_{73.5} \mathrm{Cu}_{1} \mathrm{Nb}_{3} \mathrm{Si}_{13.5} \mathrm{~B}_{9}$ layer. Its intensity increases at reversal fields below 30 Oe, and initially stabilizes its position at $\approx 4$ Oe for $H_{r}$ between -40 and -50 Oe, indicating approach of the sole soft magnetic phase

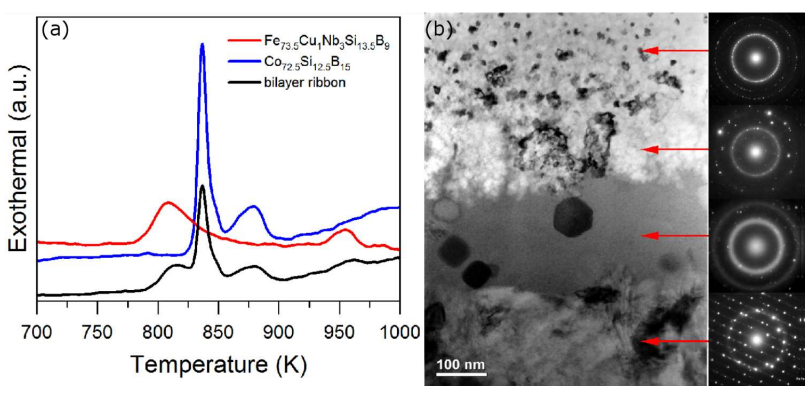

Fig. 1. (a) DSC curves of the bilayer ribbon as well as reference single-layer ribbons. (b) Cross-sectional TEM image and SAED patterns of the interlayer region in the annealed bilayer ribbon.

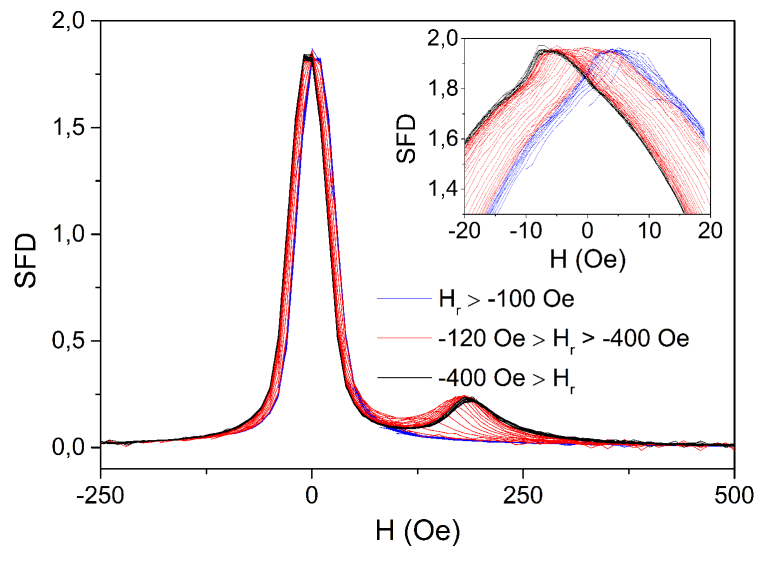

Fig. 2. Switching field distribution (SFD) of the annealed bilayer ribbon. Inset depicts detail of the SFD in the low magnetic field region.

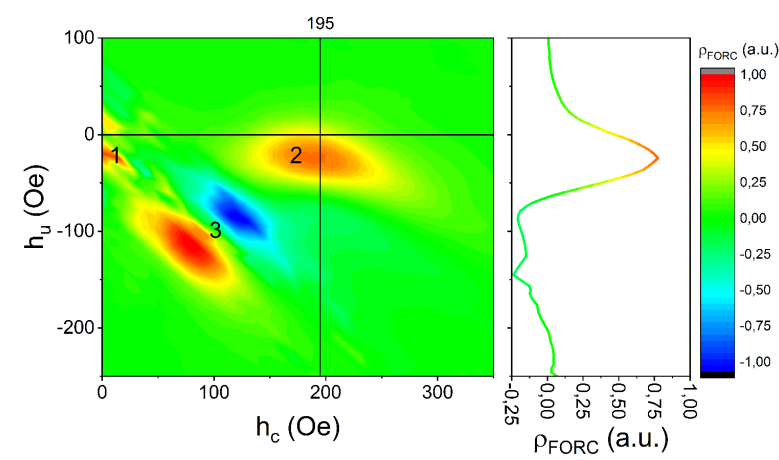

Fig. 3. Normalized FORC distribution of the annealed bilayer ribbon. Profile graph depicts $\rho_{\text {FORC }}$ projected onto $h_{u}$ axis at $h_{c}=195$ Oe..

to its magnetic saturation. This magnetic phase is represented by region 1 in the contour plot of $\rho_{\text {FORC }}$ in Fig. 3. Irreversible magnetization switching in the semihard magnetic $\mathrm{Co}_{72.5} \mathrm{Si}_{12.5} \mathrm{~B}_{15}$ layer emerged below $H_{r} \approx-120$ Oe. Intensity of the SFD peak increased with decreasing reversal field and its position shifted to the more positive magnetic field values, implying presence of exchange coupling within individual grains of the respective phase [12]. This is supported by position of respective region 2 in the contour plot of $\rho_{\text {FORC }}$ which is shifted towards negative values of interaction field $h_{u}=\frac{1}{2}\left(H+H_{r}\right)$ [13]. Mean local interaction field in this ferromagnetic phase was estimated to $H_{\text {int }} \approx 52$ Oe from vertical full width at half maximum (FWHM) of $\rho_{\mathrm{FORC}}$ projected onto $h_{u}$ axis [14-16], see Fig. 3. At the same time, progressive shift of the SFD peak representing the soft magnetic layer to lower and even negative values of magnetic field $H$ has been recognized. Additional twin contour plot of $\rho_{\text {FORC }}$ (region 3 ) suggests presence of some interlayer interaction of the magnetically harder phase upon the softer one, as derived theoretically in [17]. Due to positive-negative ordering of the values it was assumed to be dipolar field originating in the $\mathrm{Co}_{72.5} \mathrm{Si}_{12.5} \mathrm{~B}_{15}$ layer altering magnetization 


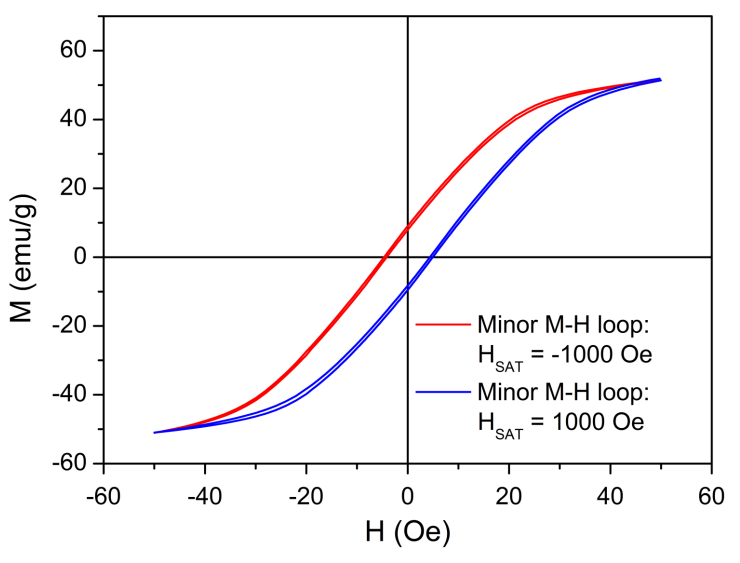

Fig. 4. Shift of the low field hysteresis loop corresponding to the soft magnetic layer obtained after previous saturation of the bilayer ribbon in the magnetic field $H_{\mathrm{SAT}}= \pm 1000$ Oe.

switching in the $\mathrm{Fe}_{73.5} \mathrm{Cu}_{1} \mathrm{Nb}_{3} \mathrm{Si}_{13.5} \mathrm{~B}_{9}$ layer. This would be in agreement with the experimental observations made for the magnetically softer bilayer composition based on FINEMET-type alloys [18].

To confirm this assumption, shift of the low field hysteresis loops corresponding to the soft magnetic Fe-based layer has been examined. Bilayer sample was foremost saturated in the magnetic field $H_{\mathrm{SAT}}= \pm 1000$ Oe which was then reduced to zero field. Afterwards a new measurement of $M-H$ loop was conducted up to a maximum field of \pm 50 Oe. By this means $\mathrm{Co}_{72.5} \mathrm{Si}_{12.5} \mathrm{~B}_{15}$ layer remained in the magnetized state close to its (positive/negative) remanence while only the magnetization of the soft magnetic $\mathrm{Fe}_{73.5} \mathrm{Cu}_{1} \mathrm{Nb}_{3} \mathrm{Si}_{13.5} \mathrm{~B}_{9}$ layer was altered by the applied magnetic field. Figure 4 shows that hysteresis loop was shifted by a bias field of 4.5 Oe to more positive $\left(H_{\mathrm{SAT}}=1000\right.$ Oe, blue loop $)$, or more negative values $\left(H_{\mathrm{SAT}}=-1000\right.$ Oe, red loop) from the origin. The dipolar field originating in the $\mathrm{Co}_{72.5} \mathrm{Si}_{12.5} \mathrm{~B}_{15}$ layer therefore plays a major role in the magnetization switching behavior in the $\mathrm{Fe}_{73.5} \mathrm{Cu}_{1} \mathrm{Nb}_{3} \mathrm{Si}_{13.5} \mathrm{~B}_{9}$ layer. Character of the shift of the low field hysteresis loop will depend on the direction and magnitude the magnetic field applied upon the bilayer sample.

\section{Conclusion}

Analysis of the magnetization reversal processes and interlayer interactions was conducted on the $\mathrm{Fe}_{73.5} \mathrm{Cu}_{1} \mathrm{Nb}_{3} \mathrm{Si}_{13.5} \mathrm{~B}_{9} / \mathrm{Co}_{72.5} \mathrm{Si}_{12.5} \mathrm{~B}_{15}$ bilayer ribbon. Annealing at $843 \mathrm{~K}$ led to development of nano/ microcrystalline structure in individual layers, which resulted in formation of two-phase soft/semihard magnetic system. Calculated minor SFD curves and FORC distribution unveiled significant influence of the dipolar field originating in the semihard magnetic Co-based layer on the hysteresis behavior of the soft magnetic Fe-based layer. Its impact was demonstrated by shift of the low field hysteresis loop after bringing semihard magnetic phase into state close to its remanence. Under such conditions maximum bias field was 4.5 Oe. The observed effect can be of potential interest for magnetic sensor applications.

\section{Acknowledgments}

This work was supported by the projects APVV-150621, VEGA 2/0171/19, ITMS 26220220186 (PROMATECH) and SAV-TUBITAK JRP grant MAGSAT.

\section{References}

[1] P. Duhaj, P. Švec, E. Majkova, V. Boháč, I. Mat'ko, Mater. Sci. Eng. A 133, 662 (1991).

[2] P. Švec, P. Švec sr., I. Maťko, I. Škorvánek, J. Kováč, D. Janičkovič, G. Vlasák, Solid State Phenom. 953, 172 (2011).

[3] L. Gonzales-Legarreta, F. Andrejka, J. Marcin, M. Varga, D. Janičkovič, P. Švec sr., I. Škorvánek, J. Alloys. Compd. 688, 94 (2016).

[4] P. Švec, I. Mat'ko, J. Marcin, J. Kováč, G. Vlasák, D. Janičkovič, I. Skorvánek, P. Svec sr., Acta Phys. Pol. A 118, 832 (2010).

[5] T. Schrefl, T. Shoji, M. Winklhofer, H. Oezelt, M. Yano, G. Zimanyi, J. Appl. Phys. 111, 07A728 (2012).

[6] S. Muralidhar, J. Gräfe, Y.C. Chen et al., Phys. Rev. B 95, 024413 (2017).

[7] D.A. Gilbert, J.W. Liao, B.J. Kirby, M. Winklhofer, C.H. Lai, K. Liu, Sci. Rep. 6, 32842 (2016).

[8] J.E. Davies, O. Hellwig, E.E. Fullerton, J.S. Jiang, S.D. Bader, G.T. Zimanyi, K. Liu, Appl. Phys. Lett. 86, 262503 (2005).

[9] C.R. Pike, A.P. Roberts, K.L. Verosub, J. Appl. Phys. 85, 6660 (1999).

[10] H.J. Harrison, M.F. Feinberg, Geochem. Geophy. Geosy. 9, Q05016 (2008).

[11] G. Herzer, Mater. Sci. Eng. A 133, 1 (1991).

[12] J.C. Martinez-Garcia, M. Rivas, D. Lago-Cachon, J.A. Garcia, J. Alloys Compd. 615, S276 (2014).

[13] D.A. Gilbert, G.T. Zimanyi, R.K. Dumas, M. Winklhofer, A. Gomez, E. Nasim, J.L. Vincent, K. Liu, Sci. Rep. 4, 4204 (2014).

[14] A.R. Muxworthy, D. Heslop, W. Williams, Geophys. J. Int. 158, 888 (2004).

[15] L. Alonso, T.R.F. Peixoto, D.R. Cornejo, J. Phys. D: Appl. Phys. 43, 465001 (2010).

[16] R.K. Dumas, C-P Li, I.V. Roshchin, I.K. Schuller, K. Liu, Phys. Rev. B 75, 134405 (2007).

[17] I. Panagiotopoulos, J. Magn. Magn. Mater. 323 2148 (2011).

[18] M. Rivas, J.C. Martinez-Garcia, I. Škorvánek, J. Marcin, P. Švec, P. Gorria, Appl. Phys. Let. 107, 132403 (2015). 